\title{
EFFECTIVENESS OF PILATES AND CAMELLIA SINENSIS SUPPLEMENTATION ON CARDIOMETABOLIC RISK FACTORS AND REDOX MARKERS IN POSTMENOPAUSAL WOMEN: A PLACEBO-CONTROLLED, RANDOMIZED TRIAL
}

\author{
S. Junges ${ }^{1}$, R. Dias Molina ${ }^{2}$, C. Bittencourt Jacondino ${ }^{1}$, M. Lopes Da Poian ${ }^{3}$, E. Tatsch ${ }^{4}$, \\ R. Noal Moresco ${ }^{4}$, M.G. Valle Gottlieb ${ }^{1}$
}

\begin{abstract}
Objective: To verify the effectiveness of Pilates and Camellia sinensis extract (CSE) supplementation on cardiometabolic risk factors and redox markers in postmenopausal women. Design: A placebo-controlled, randomized trial. Setting: communitydwelling postmenopausal women without disability. Participants: fifty postmenopausal women volunteers with cardiometabolic risk factors (high waist circumference, triglycerides, HDL-c, glucose and blood pressure). The volunteers participants were randomized in four groups: Pilates+CSE (14); Pilates+Placebo (11); CSE (11); and Placebo (14). Intervention: the CSE and Pilates+CSE intervention groups consumed one 500mg CSE capsule with excipient per day for 24 weeks. The Placebo group consumed one capsule with a placebo excipient per day for 24 weeks. Pilates training was performed twice weekly for 60 minutes each time, over 24 weeks. Measurements: cardiometabolic risk factors (glucose, waist circumference (WC), systolic blood pressure (SBP), diastolic blood pressure (DBP), triglycerides, high-density lipoproteins-HDL-c) and oxidative metabolism markers (advanced oxidation protein products (AOPP), ferric-reducing ability of plasma (FRAP), nitrosative stress marker (NOx), ischemia modified albumin (IMA). Results: When baseline variables were adjusted, the WC of the Pilates + CSE was significantly lower than that of the CSE and Placebo groups after the intervention $(p<0,001)$. The triglycerides levels of the Pilates + CSE and Pilates + Placebo groups were significantly lower than those of the Placebo group $(p=0,010)$. The glucose levels of the Pilates + CSE group were significantly lower than those of the Placebo group $(\mathrm{p}=0,041)$. Whitin-group pre and post intervention comparison showed that Pilates+CSE group presented the best effect in some cardiometabolic risk factors, with significant reductions in tree cardiometabolic risk factor: waist circumference, triglycerides, glucose and FRAP $(\mathrm{P}=0.003, \mathrm{P}<0.001, \mathrm{P}=0.021$ and $\mathrm{P}=0.041$, respectively). The Pilates+Placebo group was found to be effective in reducing triglycerides $(\mathrm{P}=0.002)$, while the $\mathrm{CSE}$ group presented increased post-intervention NOx levels $(\mathrm{p}=0.009)$. Conclusion: Our results suggest that Pilates and Camellia sinensis extract intervention may help to reduce some cardiometabolic risk factors in postmenopausal women.
\end{abstract}

Key words: Cardiometabolic risk factor, Pilates method, camellia sinensis, oxidants, antioxidant, women.

\section{Introduction}

Cardiovascular diseases (CVD) are the main cause of morbidity and mortality in developed countries and development, as is the case in Brazil (1). There are several cardiovascular risk factor, however due to its high morbidity burden and prevalence in populations,

1. Graduate Program in Biomedical Gerontology of the Institute of Geriatrics and Gerontology, Pontifical Catholic University of Rio Grande do Sul (PUCRS), Porto Alegre, Brazil; 2. Graduate Program in Medicine and Health Sciences, Pontifical Catholic University of Rio Grande do Sul (PUCRS), Porto Alegre, Brazil; 3. Pharmacist, Graduate of the Lutheran University of Brazil, Porto Alegre, Brazil. 4. Department of Clinical and Toxicological Analysis, Health Sciences Center, Federal University of Santa Maria, Santa Maria, Brazil.

Corresponding Author: Maria Gabriela Valle Gottlieb, Avenida Ipiranga, 6681 Prédio 81, $7^{\circ}$ andar, sala 703, CEP: 90619-900, Porto Alegre, RS, Brasil, E-mail: maria.gottlieb@pucrs.br obesity, diabetes, dyslipidemia and sedentary lifestyle stand out (1-3). Additionally, cardiometabolic risk factors are those involved in the development of type $2 \mathrm{DM}$ and CVD due to a set of modifiable risk factors (1-3). In this sense, several studies have demonstrated that sedentarism is one of the main risk factors that can contribute to the onset of metabolic disorders and CVD (4-7). In parallel, studies involving lifestyle modifications, such as adherence to a balanced and healthy diet and the practice of regular physical exercise, have presented beneficial results for both weight reduction and MetS components (8-10). The evidence for this has been demonstrated in research indicating that both physical exercise and bioactive dietary compounds are able to maintain the oxidation process within physiological limits, controlling damage to macromolecules, which 
can lead to systemic damage that is often irreversible $(8-9,11)$. In this context, the Pilates exercise method (an exercise that combines strength and endurance) has emerged as a physical exercise with high numbers of participants in several countries, focusing on postural correction and acting on the back and abdominal muscles, thus helping prevent or mitigate the risk factors for cardiovascular and metabolic diseases (8). An important principle of this method is the respiratory maneuvers technique that demands a constant energy expenditure for all movements, preventing excessive cardiac stress, which is, in turn, reflected in the hemodynamic parameters $(12,13)$. Moreover, some studies involving Pilates have demonstrated its effectiveness in improving the body composition of postmenopausal women (12, 14). Yet physical exercise alone is not enough to prevent cardiometabolic risk factor and CVD, being essential the combination of a diet rich in bioactive components. The consumption of certain functional foods rich in bioactive and antioxidant substances, such as green tea that comes from the Camellia sinensis plant, can also be a strongally, contributing to the prevention of cardiometabolic risk factors and/or MetS, as well reducing oxidative processes $(8,11,13)$. The Camellia sinensis plant presents a high content of flavonoids (catechins), which have a thermogenic effect and oxidize body fat. This can result in weight loss and decreased abdominal adiposity, and is therefore considered a powerful resource for the prevention of MetS components $(15,16)$. The antioxidant activity of green tea, other than it being rich in flavonoids, lies in its content of carotenoids, tocopherol, ascorbic acids and minerals ( $\mathrm{Zn}, \mathrm{Ca}, \mathrm{K}, \mathrm{Mn})$, which enhance its antioxidant potential. Green tea polyphenols have in vitro antioxidant activity that neutralizes reactive oxygen and nitrogen species (17). Furthermore, they have also shown an ability to chelate metals, such as iron, preventing their participation in reactions that generate free radicals like Fenton and Haber-Weiss, which are extremely harmful to lipids, proteins and DNA (17). There is a gap in knowledge about the effects and benefits of the pilates method on cardiometabolic risk factors, as well as the combination of the method with functional foods such as camellia sinensis. Based on this evidence, this study aimed to verify the effectiveness of the Pilates training method and the use of Camellia sinensis extract (CSE) on cardiometabolic risk factors and redox markers in postmenopausal women.

\section{Materials and methods}

\section{Study design}

\section{A placebo-controlled, randomized clinical trial}

Population and sample: The population investigated were community-dwelling postmenopausal women without disability living in Porto Alegre, the capital city of Rio Grande do Sul state, Brazil. All women had a cardiometabolic risk factors and no history of cardiovascular event at the start of the study. Based on previous studies the initial sample size was 60 women, culminating in a final sample of 50 volunteers $(9,12,18)$. The research was submitted to and approved by the Ethics Committee of the Pontifical Catholic University of Rio Grande do Sul (protocol n474.390). Data collection began after approval and complied with requirements set out in Resolution 466/2012 of the Ministry of Health in relation to research involving humans. All participants signed the Consent Form. The project was sent to the Brazilian Clinical Trials Registry (ReBEC) and registered under number RBR-2sgtn2.

\section{Recruitment}

The recruitment campaign for community-dwelling post menopausal women with cardiometabolic risk factors was conducted via phone book, newspapers and were distributed of leaflets in pharmacies, health posts and supermarkets invited for the study. All women who presented clinical exams and a declared use of medications for diabetes, hypertension or dyslipidemia at the time of recruitment were considered to have cardiometabolic risk factors and were invited to participate in the study. After clarification of aspects related to the study, initial exams presented by the participants were analyzed in order to evaluate the cardiometabolic risk factors or MetS diagnosis, according to diagnostic criteria set by the National Cholesterol Education Program (NCEP), Expert Panel on detection, evaluation and treatment of high blood cholesterol in adults (Adult Treatment Panel) III NECP-ATPIII, of 2001 (2). After selection, volunteers were scheduled for blood collection and biochemical analysis to confirm the presence of the cardiometabolic risk factors or MetS components and to obtain anthropometric variables. The procedures for fasting blood collection, anthropometric measurements and blood pressure evaluation were explained to the participants and they were asked to sign the study Informed Consent Form. The inclusion criteria comprised: being in menopause for more than a year; and have cardiometabolic risk factors based on the NCEP-ATPIII (2001) criteria which include: altered WC: $>102 \mathrm{~cm}$ to man and $>88 \mathrm{~cm}$ (women), or dyslipidemia (TG $\geq 150 \mathrm{mg} / \mathrm{dl}$ and HDL $<40 \mathrm{mg} / \mathrm{dl}$ (man), $<50 \mathrm{mg} /$ dl (women); or systolic blood pressure $\geq 130 \mathrm{mmHg}$ or diastolic blood pressure $\geq 85 \mathrm{mmHg}$ (or the use of antihypertensive medication); and/or fasting glycemia $\geq 110 \mathrm{mg} / \mathrm{dl}(2)$. Women were excluded from the study if they reported previous cardiovascular events (acute myocardial infarction, stroke, angina, heart failure), suffered from liver, gastric or thyroid diseases, presented memory problems, anxiety or sleep disorders, had any physical condition that might make Pilates training 
impossible, had undertaken Pilates or any other form of regular physical exercise, habitually or regularly drink green tea, or took vitamin supplements.

\section{Randomization}

All women with cardiometabolic risk factors were evaluated for exclusion criteria and those eligible were randomized, as shown in Figure 1. Randomization was performed through one-by-one drawing, using a box from which numbers corresponding to the women were randomly selected. In this way, the women were randomly divided into one of four groups: 1) Pilates+CSE $(\mathrm{n}=14) ; 2)$ Pilates+Placebo $(\mathrm{n}=11) ; 3) \operatorname{CSE}(\mathrm{n}=11) ; 4)$ Placebo $(n=14)$.

\section{Figure 1}

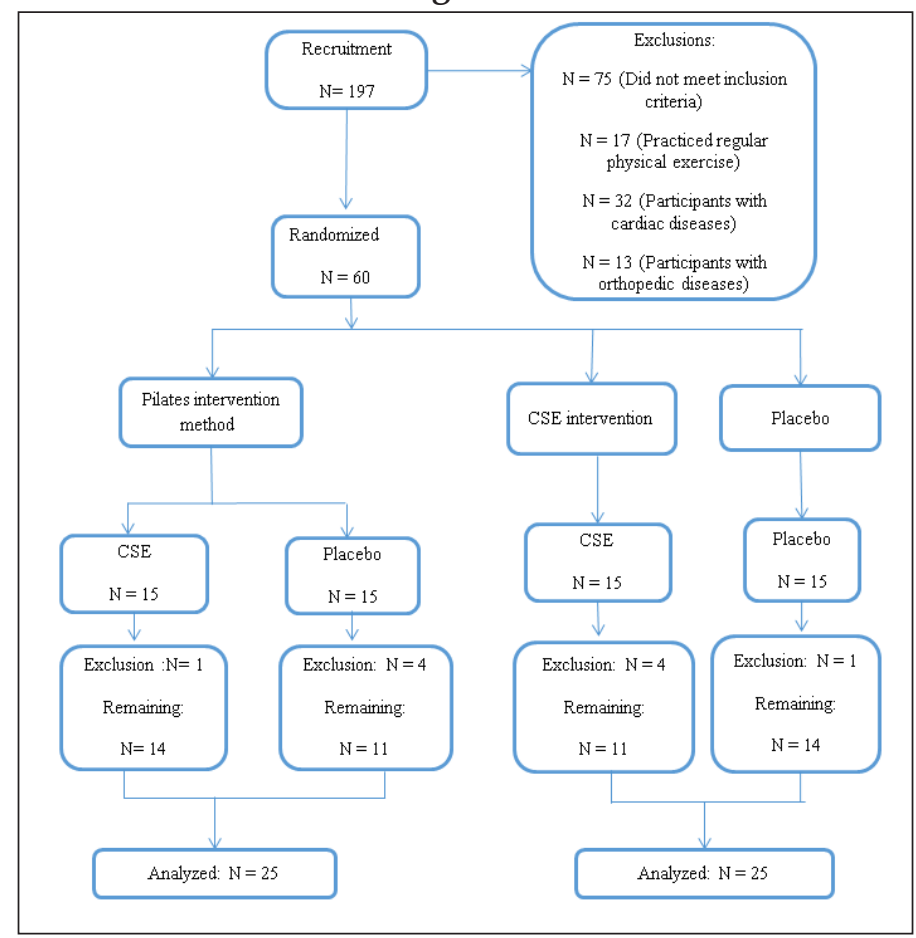

\section{Intervention}

Camellia sinensis extract and Placebo. Both the placebo and Camellia sinensis extract capsules were of the same size, raw material quantity (500 mg) and color, and were prepared in the same batch acquired from the manipulation pharmacy, Dapelle Ltd. Each placebo capsule contained 1.0000 q.s corn starch, $0.5 \%$ magnesium stearate, $0.5 \%$ Aerosil (pharmaceutical talc, light magnesium carbonate, light magnesium oxide, talc, dibasic calcium phosphate, etc.) and 29\% microcrystalline cellulose, and contained no detectable levels of polyphenol ingredients. Each CSE capsule had $500 \mathrm{mg}$ dry extract of Camellia sinensis, with a total of $4.24 \%$ polyphenols, $3.60 \%$ tannin and $0.70 \%$ caffeine. The Pilates+CSE and Pilates+Placebo intervention group participants took one capsule containing $500 \mathrm{mg}$ CSE or placebo per day, one hour after the main meal, for 24 consecutive weeks.

\section{Training protocol}

Pilates exercises were performed and completed by 25 randomized women in the following groups: Pilates+CSE $(n=14)$ and Pilates+Palcebo $(n=11)$. The intervention lasted 24 weeks, with two 60 min sessions per week. Exercises were conducted using specialized equipment: Reformer, Cadillac, Wall unit, Ladder Barrel and Chair; and floor exercises, which were classified as being of a basic level. All exercises were performed in a progressive and educational form, respecting the fundamental principles of the Pilates method. Training was divided into initial, main and final phases, consisting of four macrocycles. All training sessions began with a $10 \mathrm{~min}$ warm-up on an ergometric treadmill (Movement RT 150 pro), at a speed of $5.0 \mathrm{Km} / \mathrm{h}$. The first four-week macrocycle was composed of educational exercises at a basic level aimed at promoting neural adaptation and an introduction of exercise for breathing techniques. The second macrocycle of eight weeks included specific strengthening exercises of the spinal column extensors, serratus, serratus anterior, abdominals, quadriceps (vastus medialis) and rotator cuff, and stretching of the pectoral (major and minor), rhomboid, trapezius, triceps, iliopsoas, hamstring and adductor muscles. The third macrocycle also lasted eight weeks, with performance of the same series of exercises but with increased spring resistance. The fourth macrocycle included variation of joint angles and distances with maintenance of load and exercise series, and ending with relaxation techniques. In addition to these, stretching exercises for the shortened muscle groups were carried out using a "tonic-ball" and "Franklin Ball".

\section{Blood collection}

Blood sample (8-hour fasting) collections were performed on two different occasions (pre- and postintervention). The samples were acquired via direct peripheral venipuncture using silicone-coated vacutainer blood collection tubes (Becton-Dickinson) containing $0.5 \mathrm{ml}$ EDTA solution, with gel and a 19G $(25 \times 10)$ disposable needle, in the cubital fossa and under aseptic conditions. All sharps materials were disposed off in special containers and the biological waste discarded in white bags. All samples were sent to the Laboratory of Biochemistry, Molecular Genetics and Parasitology of the Institute of Geriatrics and Gerontology-PUCRS for due processing (separation of plasma, serum and buffy coat), and were then stored in a freezer at $-20^{\circ} \mathrm{C}$.

\section{Blinding}

All researchers involved were blinded throughout this study. However, the pharmacist who distributed the CSE 
and placebo capsules, the Pilates group participants and instructors were not blinded for the Pilates training.

\section{Evaluation of study adherence}

The concept of adherence varies, but in general, a participant is understood to be adherent to a therapy using prescribed medications or other procedures when they observe the schedules, dosages and treatment durations for at least $80 \%$ of the total protocol. To evaluate the percentage of participant adherence in the Placebo, CSE and Pilates+CSE groups, participants were requested to present those capsules that were not consumed during the intervention period. Women who consumed less than $80 \%$ of capsules, together with those who failed to attend at least $80 \%$ of the Pilates training sessions, were excluded from the study $(16,18)$. Four women ( 1 from the Pilates + CSE group and 4 from the Pilates + placebo group) were excluded from the study because they had a frequency of less than $80 \%$ in Pilates classes and/or in the use of the capsules. In addition, five women (4 CSE and 1 from placebo group) were excluded because they dropped out.

\section{Evaluation of Adverse Effects}

The study participants were asked to report adverse effects at any time in relation to the placebo, green tea or Pilates. None of the participants from any of the groups observed in this study complained of any adverse effects.

\section{Variables and collection tools}

The following variables were investigated for all study participants: age, marital status, education and income. Data were collected through use of a structured questionnaire. The cardiometabolic risk factors, such as fasting glucose and lipid profile, were measured using Labtest kits and analyzed by spectrophotometry, in accordance with manufacturer instructions. Weight was measured using a calibrated anthropometric balance scale with a $150 \mathrm{~kg}$ capacity, graduated in $100 \mathrm{~g}$ increments. Participants were weighed barefoot wearing a bathing suit only, with results recorded in kilograms (kg). Participant height was recorded using the measuring rod of the scales. Body Mass Index was then calculated through the equation, weight $(\mathrm{kg})$ divided by height $(\mathrm{m})$ squared. The minimum abdominal or waist circumference (WC) was recorded at the level of the iliac crest and values up to $80 \mathrm{~cm}$ were considered normal for women. Above this value, individuals were considered to have central obesity (2). Evaluation of the levels of advanced oxidation protein products (AOPP) was performed according to Witko-Sarsat et al. (19), in a Cobas MIRA® analyzer (Roche Diagnostics, Basel, Switzerland). Nitrosative stress (NOx) were assessed based on the technique described by Tatsch et al. (20), in a Cobas MIRA ${ }^{\circledR}$ analyzer (Roche Diagnostics, Basel, Switzerland). The ferric reducing ability of plasma (FRAP) was performed following the technique described by Benzie et al. (21), in a Cobas MIRA ${ }^{\circledR}$ analyzer (Roche Diagnostics, Basel, Switzerland). Ischemia modified albumin (IMA) was measured by colorimetric assay with cobalt, described by Bar-Or et al. (22).

A nurse measured arterial blood pressure, recording systolic blood pressure (SBP) and diastolic blood pressure (DBP) using a mercury sphygmomanometer (Erka, Germany), equipped with an appropriately sized cuff for the right arm circumference.

Each participant remained at rest (sitting) for at least $5 \mathrm{~min}$ before the measurement was taken. Two measurements were recorded with an approximate interval of $30 \mathrm{~min}$ between them. The appearance of sounds was used for identification of SBP and their disappearance (Korotkoff phase V) for DBP. The Osler maneuver for measurement of systolic arterial blood pressure (considered positive when the radial artery is still palpable after inflating the cuff above the SBP level) was concomitantly used due to the possibility of pseudohypertension caused by stiffening of the brachial artery, secondary to atherosclerosis (can increase arterial blood pressure by $30 \mathrm{mmHg}$ or more). These cases were excluded from the study. Blood pressure levels up to $130 / 85 \mathrm{mmHg}$ were considered normal in accordance with recommendations from the National Cholesterol Education Program (NCEP) expert panel on detection, evaluation and treatment of high blood cholesterol in adults (Adult Treatment Panel III) (2). Diagnosis of MetS was made according to the NCEP-ATP III diagnostic criteria (2).

\section{Statistical Analysis}

Data were recorded using an Excel spreadsheet and analyzed through the IBM statistics package, SPSS, version 21.0. Continuous variables were expressed as mean and standard deviation/standard error or median and interquartile range. Categorical variables were described by absolute and relative frequencies. One-way analysis of variance (ANOVA) was applied followed by Tukey's post hoc test to compare the mean values. The Kruskal-Wallis test was used in the case of asymmetry. The Analysis of Covariance (ANCOVA), complemented by the Bonferroni test, was applied to adjust for differences concerning baseline measurements. Intragroup comparisons were evaluated using Student's $t$ test for paired samples (symmetrical distribution) or the Wilcoxon test (asymmetric distribution). A significance level of $5 \%$ was adopted $(\mathrm{p}<0.05)$. 
Table 1

Comparison of cardiometabolic risk factors $(\mathrm{CRF})$ and redox markers between the groups in the pre-intervention period

\begin{tabular}{|c|c|c|c|c|c|}
\hline Variables & $\begin{array}{c}\text { Pilates+CSE } \\
(\mathbf{n}=14) \\
\text { Mean } \pm \text { SD }\end{array}$ & $\begin{array}{c}\text { Pilates+Placebo } \\
(\mathbf{n}=\mathbf{1 1}) \\
\text { Mean } \pm \text { SD }\end{array}$ & $\begin{array}{c}\text { CSE } \\
(n=11) \\
\text { Mean } \pm S D\end{array}$ & $\begin{array}{c}\text { Placebo } \\
(\mathrm{n}=14) \\
\text { Mean } \pm \text { SD }\end{array}$ & $\mathrm{p}$ \\
\hline \multicolumn{6}{|l|}{$C R F$} \\
\hline WC & $100.5 \pm 11.7$ & $95.5 \pm 9.3$ & $100.9 \pm 11.7$ & $105.5 \pm 6.5$ & 0.114 \\
\hline SBP & $125.7 \pm 9.4$ & $120.0 \pm 14.1$ & $126.4 \pm 13.6$ & $122.9 \pm 19.0$ & 0.707 \\
\hline DBP & $80.0 \pm 9.6$ & $77.3 \pm 10.1$ & $77.3 \pm 10.1$ & $76.4 \pm 10.1$ & 0.799 \\
\hline TG & $187.1 \pm 61.7^{b}$ & $154 \pm 42.7^{\mathrm{b}}$ & $100.7 \pm 31.2^{\mathrm{a}}$ & $154.1 \pm 49.5^{\mathrm{b}}$ & 0.001 \\
\hline HDL & $44.6 \pm 9.7$ & $41.9 \pm 7.1$ & $48.5 \pm 11.1$ & $46.6 \pm 9.0$ & 0.397 \\
\hline Blood glucose & $152.7 \pm 66.9$ & $119.8 \pm 33.7$ & $112.8 \pm 24.5$ & $113.7 \pm 45.8$ & 0.106 \\
\hline \multicolumn{6}{|l|}{ Redox markers } \\
\hline $\mathrm{AOPP}^{*}$ & $56,9(36,9-71,1)$ & $57.2(45.9-77.4)$ & $69.1(43.2-83.8)$ & $51.2(28.7-81.5)$ & 0.869 \\
\hline FRAP $^{*}$ & $519(308-639)$ & $423(394-477)$ & $513(261-639)$ & $497(248-594)$ & 0.775 \\
\hline $\mathrm{NOx} *$ & $122(89-323)$ & $125(74-182)$ & $96(64-159)$ & $125(98-177)$ & 0.511 \\
\hline IMA & $0.58 \pm 0.09$ & $0.58 \pm 0.25$ & $0.53 \pm 0.18$ & $0.61 \pm 0.18$ & 0.846 \\
\hline
\end{tabular}

\section{Results}

The sample comprised of 50 volunteers with a cardiometabolic risk factors, with a mean age of $61.3 \pm 6.6$ years (range 51-77 yr). The mean age did not differ between the groups, being respectively: Pilates + CSE group (60.5 $\pm 6.2 \mathrm{yr})$, Pilates + Placebo group (62.6 $\pm 6.7 \mathrm{yr})$, CSE group $(59.1 \pm 4.7 \mathrm{yr})$ and Placebo group (62.9 \pm 8.0 yr), with $\mathrm{p}=0.462$. No statistically significant differences between the investigated groups were observed for the sociodemographic variables of race, income and education ( $p>0.05)$.

Investigation of the components of MetS for the 50 study participants revealed that $35(70 \%)$ were verified as presenting three MetS diagnostic criteria components and $15(30 \%)$ presented two (NCEP ATP III). All participants recorded a waist circumference $>88 \mathrm{~cm}$. A comparison was made between the initial measurement results for the four groups in all the recorded variables (Table 1). The triglyceride (TG) levels for those patients from the Camellia sinensis group were observed to be significantly lower than the other groups. No statistically significant differences between the groups were seen for the remaining variables before the intervention began.

The post-intervention variables between the four groups are compared in Table 2. After adjustment for the baseline measurements, the post-intervention WC variable for the Pilates+CSE group was significantly lower than the CSE and Placebo groups.

The TG levels for the Pilates+CSE and Pilates+Placebo groups were significantly lower than the Placebo group. Blood glucose for the Pilates+CSE group were significantly lower than the Placebo group. No statistically significant differences were observed in the redox markers.

Table 3 compares intragroup variables. A significant reduction in WC, TG, blood glucose levels and FRAP was found in the Pilates+CSE group. In the Pilates+Placebo group, a significant reduction was seen only in the TG variable. A significant increase in NOx levels was verified in the CSE group, while in the Placebo group a significant increase in WC and FRAP and significant reduction of triglycerides was observed.

\section{Discussion}

This study verified the effectiveness of Pilates training and the use of Camellia sinensis extract on the cardiometabolic risk factors of postmenopausal women. The results suggested that the combination of Pilates+CSE can be the most effective method for reducing three important cardiometabolic risk factor, being waist circumference, triglycerides and blood glucose, and for increasing levels of FRAP. In addition, supplementation with CSE alone suggested increase NOx levels. Furthermore, the Pilates+Placebo combination also seemed to be effective in reducing triglyceride levels. Researches on this topic are relatively recent and yet inconclusive, especially in relation to the use of Pilates training. Most studies focused on this method 
Table 2

Comparison between groups of cardiometabolic risk factors (CRF) and redox markers in the post-intervention period

\begin{tabular}{|c|c|c|c|c|c|}
\hline Variables & $\begin{array}{c}\text { Pilates+CSE } \\
(n=14) \\
\text { Mean } \pm \text { SE }\end{array}$ & $\begin{array}{c}\text { Pilates+Placebo } \\
(\mathbf{n}=\mathbf{1 1}) \\
\text { Mean } \pm \text { SE }\end{array}$ & $\begin{array}{c}\text { CSE } \\
(n=11) \\
\text { Mean } \pm S E\end{array}$ & $\begin{array}{c}\text { Placebo } \\
(n=14) \\
\text { Mean } \pm \text { SE }\end{array}$ & adjustedp* \\
\hline \multicolumn{6}{|l|}{$C R F$} \\
\hline WC & $96.8 \pm 0.94^{a}$ & $99.6 \pm 1.10^{\mathrm{ab}}$ & $101.4 \pm 1.06^{\mathrm{b}}$ & $103 \pm 0.97^{b}$ & $<0.001$ \\
\hline SBP & $121.4 \pm 1.6$ & $123.7 \pm 1.8$ & $125.4 \pm 1.8$ & $127.1 \pm 1.6$ & 0.086 \\
\hline DBP & $79.0 \pm 1.3$ & $77.7 \pm 1.4$ & $77.7 \pm 1.4$ & $77.4 \pm 1.3$ & 0.819 \\
\hline TG & $92.6 \pm 3.4^{\mathrm{a}}$ & $99.4 \pm 3.6^{\mathrm{a}}$ & $98.9 \pm 4.0^{\mathrm{ab}}$ & $107.6 \pm 3.2^{\mathrm{b}}$ & 0.010 \\
\hline HDL & $45.4 \pm 2.3$ & $49.7 \pm 2.7$ & $42.8 \pm 2.7$ & $40.2 \pm 2.3$ & 0.074 \\
\hline Blood glucose & $101 \pm 13.1^{\mathrm{a}}$ & $117.9 \pm 14.1^{\mathrm{ab}}$ & $135.8 \pm 14.2^{\mathrm{ab}}$ & $154.0 \pm 12.6^{\mathrm{b}}$ & 0.041 \\
\hline \multicolumn{6}{|l|}{ Redox markers } \\
\hline $\mathrm{AOPP}^{* *}$ & $68.8 \pm 5.3$ & $71.7 \pm 5.5$ & $74.2 \pm 5.8$ & $75.2 \pm 4.9$ & 0.853 \\
\hline FRAP** & $703.5 \pm 67.5$ & $627.2 \pm 69.8$ & $595.6 \pm 73.3$ & $787.1 \pm 62.1$ & 0.155 \\
\hline $\mathrm{NOx}^{* *}$ & $207.2 \pm 28.4$ & $180.9 \pm 29.1$ & $191.7 \pm 31.1$ & $182.9 \pm 36.5$ & 0.946 \\
\hline IMA & $0.50 \pm 0.06$ & $0.45 \pm 0.06$ & $0.54 \pm 0.07$ & $0.64 \pm 0.07$ & 0.240 \\
\hline
\end{tabular}

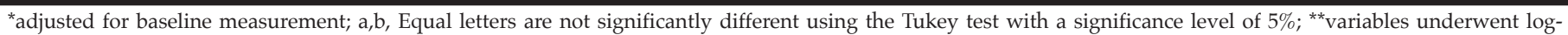
transformation for conducting ANOVA.CRF: cardiometabolic risk factors; CSE: Camellia sinensis extract; WC: waist circumference; SBP: systolic blood pressure; DBP:

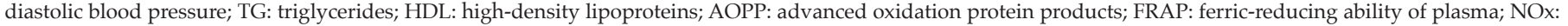
nitrosative stress marker; IMA: ischemia modified albumin.

have investigated its effectiveness in chronic low back pain, postural correction and improvement of dynamic balance, flexibility, strength and endurance, increasing muscle mass and reducing fat mass, rehabilitation, and improvements in quality of life $(12,14,18,23)$. The effects of Pilates on cardiometabolic risk factors has been investigated more recently, however, the literature is still scarce $(8,9,24)$. Studies are more abundant in relation to the consumption of Camellia sinensis extract, with reports on its beneficial effects on adipogenesis, mitigation of MetS, particularly with regard to hyperglycemia, hypercholesterolemia, and waist circumference, as well as in lessening oxidative stress $(11,15,16)$. These effects have been observed in human and nonhuman experimental models, and for its consumption both in the form of tea (infusion) and capsules (extract).

Pilates combined with CSE supplementation presented better efficacy in mitigating three components of MetS that have a high level of morbidity and mortality (waist circumference, blood levels of triglyceride and glucose) in postmenopausal women. Double-blind randomized clinical trials, such as the Heart Outcomes Prevention Evaluation (HOPE), have already shown that obesity, especially abdominal, worsens the prognosis of patients with cardiovascular diseases. This study demonstrated that abdominal obesity alone is associated with a $20-23 \%$ increase in relative risk for myocardial infarction, $38 \%$ for heart failure, and $17 \%$ for total mortality (25). Abdominal obesity, which is metabolically active, produces chronic low-grade inflammation, increased flow of free fatty acids and an increase in LDL-c plasma levels (26). The union of Pilates and CSE can promote a better distribution of adipose tissue through increased mobilization of fat deposited in the abdominal region $(12,14,15,27)$. In addition, studies have demonstrated that catechins (EGCG) of Camellia sinensis promote weight loss, affect fat distribution, inhibit adipogenesis and cause apoptosis in mature adipocytes $(12,14,15,27)$. The findings of the present study reinforce the hypothesis that Pilates exercises involve a considerable energy expenditure through the performance of multidimensional and resistance exercises, which are of low and moderate intensity, contributing to the burning of lipids and preventing or decreasing their accumulation in adipose tissue (28). This in turn may contribute to the prevention of cardiovascular diseases $(8,9,28)$. One study analyzed the effect of Pilates on a diabetic group but found no statistical difference in the mean glycated hemoglobin and daily doses of subcutaneous insulin between the intervention and control groups (24). A hypothesis proposed by the authors suggested that participants increased their carbohydrate intake prior to the physical activity due to the risk of hypoglycemia while performing the exercise. However, their caloric intake was not evaluated (24). In relation to lipid profile, no statistically significant differences were found in the mean levels of triglycerides, HDL-c, LDL-c and total cholesterol (24) in the Pilates intervention group. Nonetheless, the findings of the present study demonstrated that both Pilates+CSE (reduction of three components of MetS) and Pilates+Placebo were able to reduce at least one of the diagnostic criterion of MetS. 
Table 3

Intragroup comparison of cardiometabolic risk factors (CRF) and redox markers, pre- and post-intervention

\begin{tabular}{|c|c|c|c|c|c|c|}
\hline \multirow[t]{2}{*}{ Variables } & \multicolumn{3}{|c|}{ Pilates+CSE $(n=14)$} & \multicolumn{3}{|c|}{ Pilates+Placebo $(n=11)$} \\
\hline & $\begin{array}{c}\text { Pre- } \\
\text { Mean } \pm \text { SD }\end{array}$ & $\begin{array}{c}\text { Post- } \\
\text { Mean } \pm S D\end{array}$ & $\mathbf{p}$ & $\begin{array}{c}\text { Pre- } \\
\text { Mean } \pm S D\end{array}$ & $\begin{array}{c}\text { Post- } \\
\text { Mean } \pm \text { SD }\end{array}$ & $\mathbf{p}$ \\
\hline \multicolumn{7}{|l|}{ CRF } \\
\hline WC & $100.5 \pm 11.7$ & $96.4 \pm 10.9$ & 0.003 & $95.5 \pm 9.3$ & $94.5 \pm 10.6$ & 0.295 \\
\hline SBP & $125.7 \pm 9.4$ & $122.9 \pm 4.7$ & 0.165 & $120.0 \pm 14.1$ & $120.9 \pm 7.0$ & 0.756 \\
\hline DBP & $80.0 \pm 9.6$ & $80.7 \pm 7.3$ & 0.720 & $77.3 \pm 10.1$ & $77.3 \pm 7.9$ & 1.000 \\
\hline TG & $187.1 \pm 61.7$ & $92.9 \pm 12.5$ & $<0.001$ & $153.9 \pm 42.7$ & $94.5 \pm 10.3$ & 0.002 \\
\hline HDL & $44.6 \pm 9.7$ & $45.1 \pm 10.1$ & 0.770 & $41.9 \pm 7.1$ & $48.7 \pm 9.2$ & 0.078 \\
\hline Blood glucose & $152.7 \pm 66.9$ & $109.9 \pm 32.9$ & 0.021 & $119.8 \pm 33.7$ & $115.9 \pm 34.2$ & 0.690 \\
\hline \multicolumn{7}{|l|}{ Redox markers } \\
\hline AOPP* & $56.9(36.9-71.1)$ & $70.1(62.2-76.2)$ & 0.158 & $57.2(45.9-77.4)$ & $68.3(62.6-82.2)$ & 0.182 \\
\hline FRAP* & $519(309-639)$ & 654(527-907) & 0.041 & $423(394-477)$ & $690(488-753)$ & 0.075 \\
\hline NOx* & $122.4(88.9-323)$ & 184.9(116-357) & 0.433 & $125.4(73.5-182)$ & 209 (113-227) & 0.328 \\
\hline IMA & $0.58 \pm 0.10$ & $0.49 \pm 0.18$ & 0.199 & $0.55 \pm 0.25$ & $0.45 \pm 0.11$ & 0.258 \\
\hline \multirow[t]{2}{*}{ Variables } & \multicolumn{3}{|c|}{$\operatorname{CSE}(n=11)$} & \multicolumn{3}{|c|}{ Placebo $(n=14)$} \\
\hline & $\begin{array}{c}\text { Pre- } \\
\text { Mean } \pm \text { SD }\end{array}$ & $\begin{array}{c}\text { Post- } \\
\text { Mean } \pm \text { SD }\end{array}$ & p & $\begin{array}{c}\text { Pre- } \\
\text { Mean } \pm S D\end{array}$ & $\begin{array}{c}\text { Post- } \\
\text { Mean } \pm \text { SD }\end{array}$ & $\mathrm{p}$ \\
\hline \multicolumn{7}{|l|}{$C R F$} \\
\hline WC & $100.9 \pm 11.7$ & $101.4 \pm 12.1$ & 0.669 & $105.5 \pm 6.5$ & $107.4 \pm 6.8$ & 0.022 \\
\hline SBP & $126.4 \pm 13.6$ & $127.3 \pm 12.7$ & 0.341 & $122.9 \pm 19.0$ & $126.4 \pm 18.6$ & 0.055 \\
\hline DBP & $77.3 \pm 10.1$ & $77.3 \pm 11.0$ & 1.000 & $76.4 \pm 10.1$ & $76.4 \pm 9.3$ & 1.000 \\
\hline TG & $100.7 \pm 31.2$ & $98.5 \pm 16.3$ & 0.828 & $154.1 \pm 49.5$ & $107.6 \pm 6.6$ & 0.005 \\
\hline HDL & $48.5 \pm 11.1$ & $43.6 \pm 5.8$ & 0.289 & $46.6 \pm 9.0$ & $40.5 \pm 9.7$ & 0.064 \\
\hline Blood glucose & $112.8 \pm 24.5$ & $131.6 \pm 54.1$ & 0.301 & $113.7 \pm 45.8$ & $150.1 \pm 64.6$ & 0.054 \\
\hline \multicolumn{7}{|l|}{ Redox markers } \\
\hline AOPP* & 69.1(43.2-83.8) & 69.7(49.3-91.3) & 0.333 & $51.2(28.7-81.5)$ & 79 (60.3-84.7) & 0.158 \\
\hline FRAP* $^{*}$ & $513(261-639)$ & 477 (409-830) & 0.333 & $496(247-593)$ & 723 (619-1004) & 0.001 \\
\hline NOx* & $96.2(64.4-160)$ & $169(112-204)$ & 0.009 & 125.2(97.9-176) & $176.1(138-231)$ & 0.310 \\
\hline IMA & $0.53 \pm 0.19$ & $0.55 \pm 0.20$ & 0.886 & $0.61 \pm 0.19$ & $0.63 \pm 0.30$ & 0.907 \\
\hline
\end{tabular}

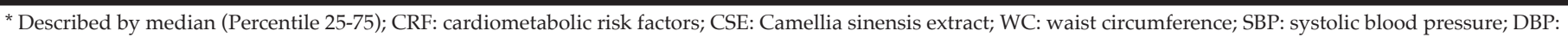

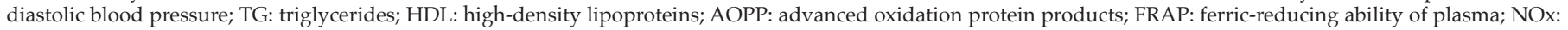
nitrosative stress marker; IMA: ischemia modified albumin

With regard to effects of intervention on the redox markers, the present study noted an increase in FRAP levels in the Pilates+CSE group and in NOx levels in the CSE group. According to Benzie et al., individuals that present high levels of FRAP have a higher antioxidant capacity in reducing iron (21). An in vitro study with CSE conducted by Sohrab et al., exhibited the ability of CSE polyphenols to chelate metals, such as iron, preventing their participation in reactions that generate free radicals like Fenton and Haber-weiss (29). Besides the antioxidant properties of CSE, according to Steinbacher and Eckl, oxidative stress induced by exercise serves as an important signal to stimulate muscle adaptation to antioxidant systems through activation of redox-sensitive signaling pathways (30). While an intense contraction is sufficient to activate these pathways, the upregulation of protein synthesis requires cumulative effects of repetitive exercise movements, which Pilates provides. This formation and adaptation also allows the cell to incorporate high levels of exogenous antioxidants in the form of dietary supplementation. This corroborates the results of the present study, in which only the Pilates+CSE group showed increases in FRAP levels. In relation to NOx findings, the bioactive components and antioxidant and anti-inflammatory activity of CSE may contribute to raise NO levels, thus increasing its 
metabolites.

Despite the beneficial results presented with respect to the cardiometabolic risk factors, it is important to consider some limitations of the present research. The first concerns is to reduced sample sizes. The second relates to the non-assessment of the macroand micronutrient intake of the sample, which could have affected the obtained results, as well as causing unexpected results in the Placebo group, especially in relation to triglycerides and FRAP. Nevertheless, despite the limitations of the present trial, our results suggest that Pilates and supplementation with Camellia sinensis decrease cardiometabolic risk factors and increase FRAP levels in postmenopausal women, which may positively influence the global health of this sample. Additionally, the study reinforces the recommendation of Pilates method exercises for this age group and a diet rich in bioactive compounds, such as the flavonoids of Camellia sinensis, not only as an adjuvant treatment, but also for the prevention cardiometabolic risk factors.

\section{Conclusion}

The presented results suggest the effectiveness of the Pilates method with CSE supplementation on cardiometabolic risk factors, such as triglycerides, blood glucose and waist circumference, as well as on FRAP. However, the Pilates+Placebo group also appear to be effective in reducing triglycerides. In addition, ingestion of CSE also appear to be effective in increasing NOx levels. Our results suggest that this type of intervention, combining Pilates and Camellia sinensis may help to reduce some cardiometabolic risk factors in postmenopausal women.

Acknowledgements: Our thanks to the National Postdoctoral Program of the Coordination for the Improvement of Higher Education Personnel (PNPD/ CAPES) and the Dapelle manipulation pharmacy for providing the extracts, and to the women who participated in the study. Trial registration: RBR-2sgtn2 (Brazilian Clinical Trials Registry- ReBEC) study.

Conflict of interest: The authors declare no conflict of interest involving this

Ethical standards: The study followed all the ethical recommendations of Resolution 466/12 of the Brazilian Ministry of Health on Research involving human beings. The investigators undertook to respect the protocol in all respects.

\section{References}

1. Brasil. Ministério da Saúde. Cadernos de Atenção Básica, no. 38, 2014. [cited 2015 March] Available at: http:/ / bvsms.saude.gov.br/bvs/publicacoes/ estrategias_cuidado_doenca_cronica_obesidade_cab38.pdf

2. National Cholesterol Education Program - NCEP. Executive Summary of the Third Report of the National Cholesterol Education Program.Expert Panel on Detection, Evaluation, and Treatment of High Blood Cholestero in Adults - ATPIII. JAMA 2001;285:2486-2497. http://dx.doi.org/10.1001/ jama.285.19.2486

3. Diretrizes da Sociedade Brasileira de Cardiologia. Pocket Book.5 $5^{a}$ edição 20112013. p.4-446.

4. González-Gross M, Meléndez A. Sedentarism, active lifestyle and sport: Impact on health and obesity prevention. Nutr Hosp. 2013;28(Suppl 5):89-98.
5. Nauman J, Nilsen TI, Wisloff U, Vatten LJ. Combined effect of resting heart rate and physical activity on ischaemic heart disease: mortality follow-up in a population study (the HUNT study, Norway). J Epidemiol Community Health. 2010;64:175-81.

6. Myers J, Prakash M, Froelicher V, Do D, Partington S, Atwood JE. Exercise capacity and mortality among men referred for exercise testing. N Engl J Med. 2002;346:793-801.

7. Aravindalochanan V1, Kumpatla S, Rengarajan M, Rajan R, Viswanathan $\mathrm{V}$. Risk of diabetes in subjects with sedentary profession and the synergistic effect of positive family history of diabetes. Diabetes Technol Ther. 2014;16(1):26-32.

8. Junges S, Jacondino CB, Gottlieb MGV. Pilates effect on risk factors for cardiometabolic disease: a systematic review. Scientia Medica. 2015;25(1): 1-8.

9. Fourie M, Gildenhuys M, Shaw I, Shaw B, Toriola A, Goon DT. Effects of a MatPilates program on cardiometabolic parameters in elderly women. Pak J MedSci. 2013; 29(2):500-4

10. Finelli C, Padula MC, Martelli G, Tarantino G. Could the improvement of obesity-related co-morbidities depend on modified gut hormones secretion? World J Gastroenterol. 2014;20(44):16649-64

11. Yousaf S, Butt MS, Suleria HAR, Iqbala MJ. The role of green tea extract and powder in mitigating metabolic syndromes with special reference to hyperglycemia and hypercholesterolemia. Food Funct. 2014;5(3):545-56.

12. Bergamin M, Gobbo S, Bullo V, et al. Effects of a Pilates exercise program on muscles trength, postural controland body composition: results from a pilot study in a group of post-menopausal women. Age (Dordr). 2015;37(6):118.

13. Qian G, Xue K, Tang L, et al. Mitigation of oxidative damage by green tea polyphenols and Tai Chi exercise in postmenopausal women with osteopenia. PLoS One. 2012;7(10)

14. Vaquero-Cristóbal R, Alacid F, Esparza-Ros F, López-Plaza D, Muyor JM, López-Miñarro PA. The effects of a reformer Pilates program on body composition and morphological characteristics in active women after a detraining period. Women Health. 2015;19:1-23.

15. Lin J, Della-Fera MA, Baile CA. Green tea polyphenol epigallocatequin Gallate inhibits adipogenesis and induces apoptosis in 3T3-L1 adipocytes. Obes Res. 2005;13(6):982-990.

16. Vieira Senger AE, Schwanke CH, Gomes I, Valle Gottlieb MG. Effect of green tea (Camellia sinensis) consumption on the components of metabolic syndromein elderly. J. Nutr Health Aging. 2012;16(9):738-42.

17. Higdon JV, Frei B. Tea catechins and polyphenols: health effects, metabolism, and antioxidant functions. Crit Rev Food Sci Nutr. 2003;43:89-143.

18. Junges S, Gottlieb MG, Babtista RR, Quadros CB, Resende TL, Gomes I. Pilates method effect for posture and flexibility in women with kyphosis. Brazilian journal of Science and Movement. 2012;20(1):21-25.

19. Witko-sarsat V, Friedlander M, Nguyen Khoa T, et al. Advanced Oxidation Protein Products as Novel Mediators of Inflammation and Monocyte Activation in Chronic Renal Failure. Journal Of Immunology. 1998; 161 (5): 2524-2532.

20. Tatsch E, Bochi, GV, Pereira RS. A simple and inexpensive automated technique for measurement of serum nitrite/nitrate. Clin Biochem. 2011; 44: 348-350.

21. Benzie IF, Strain JJ. The ferric reducing ability of plasma (FRAP) as a measure of "antioxidant power": the FRAP assay. Anal Biochem. 1996; 239 (1):70-76.

22. Bar-or D, Lau E, Winkler JV. Novel assay for cobalt-albumin binding and its potential as a marker for myocardial ischemia-a preliminary report. J Emerg Med. 2000; 19: 311-315.

23. Natour J, Cazotti L de A, Ribeiro LH, Baptista AS, Jones A. Pilates improves pain, function and quality of life in patients with chronic low back pain: a randomized controlled trial. Clin. Rehabil. 2015;29(1):59-68.

24. Tunar M, Ozen S, Gaksen D, Asar G, Bedizds S, Darcan S. The effects of Pilates on metabolic control and physical performance in adolescents with type 1 diabetes mellitus. J. Diabetes Complications. 2012;26(4):348-51.

25. Dagenais GR, Yi Q, Mann JF, Bosch J, Pogue J, Yusuf S. Prognostic impact of body weight and abdominal obesity in women and men with cardiovascular disease. Am Heart J. 2005;149(1):54-60.

26. Boden G. Obesity and Free Fatty Acids (FFA). Endocrinol Metab Clin North Am. 2008; 37(3): 635-ix.

27. Lin JK, Lin-Shiau SY. Mechanismis of hypolipidemic and anti-obesity effects of tea and tea polyphenols. Mol Nutr Food Res. 2006;50:211-7.

28. Torabian M, Taghadosi M, Ajorpaz NM, Khorasanifar L. The effect of Pilates exercises on general in women with type 2 diabetes. Life Science Journal .2013;10: 283-288

29. Sohrab G; Hosseinpour-Niazi S, Hejazi J, Yuzbashian E, Mirmiran P, Azizi F. Dietary polyphenols and metabolic syndrome among Iranian adults. Int J Food Sci Nutr. 2013; 64 (6): 661-667.

30. Steinbacher P, Eckl P. Impact of oxidative stress on exercising skeletal muscle. Biomolecules. 2015;5(2):356-77. 